\title{
Coral compound fights neuropathic pain
}

Neuropathic pain is chronic pain that can arise from nerve damage resulting from inflammation in the central nervous system (CNS). People worldwide suffer from neuropathic pain, which can include hyperalgesia (heightened pain sensation, such that mildly uncomfortable stimuli are perceived as severely painful) and allodynia ('misplaced' pain, such that nonpainful stimuli are perceived as painful). The few available treatments for neuropathic pain are relatively ineffective and have potentially dangerous side effects; safe, effective options are badly needed.

Zhi-Hong Wen, Yen-Hsuan Jean and colleagues (Department of Marine Biotechnology and Resources, National Sun Yat-Sen University, Taiwan) may be close to providing one. They have discovered that a compound called capnellene (named after the soft coral from which it is isolated, Capnella imbricata) has pain-relieving effects in a rat model of neuropathic pain.

Wen's group tested two capnellene compounds in male Wistar rats with chronic constriction injury, an

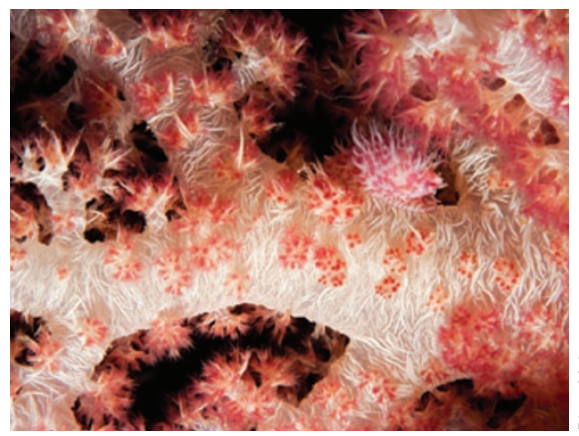

a single intraperitoneal dose; this route was also effective for pain relief, taking effect within 1-3 h and lasting for at least $6 \mathrm{~h}$. Administration of capnellene did not cause any neurological problems in normal rats.

Capnellene's analgesic effects may stem from its inhibition of the expression of COX-2 protein in the spinal cord. COX-2 is a pro-inflammatory protein that can excite the nerves that carry pain signals. It is released by cells surrounding nerve cells, experimental model of neuropathic pain. The compounds were administered intrathecally and intraperitoneally, and researchers assessed the rats' thermal hyperalgesia response by measuring the time they took to withdraw a hind paw from a low-intensity heat source ( $\mathrm{Br}$. J. Pharmacol. published online 5 August 2009; doi:10.1111/j.1476-5381.2009.00323.x). After intrathecal administration, both compounds acted quickly to relieve pain. Pain relief was dose-dependent, reached a maximum about 30 min after compounds were administered and lasted 30-180 min. One of the compounds was also tested as such as microglia, when they are activated by some disturbance to the CNS. In Wen's study, both capnellene compounds inhibited expression of COX-2 in activated microglial cells in vitro.

The research results suggest that capnellene could have clinical application as a treatment for neuropathic pain. "Today there are few pharmacological agents that can help people suffering from neuropathic pain, but we believe that these marine-derived compounds could lead to the development of a new range of drugs of great potential," stated Wen.

Monica Harrington

\section{BLUE DYE BLOCKS DAMAGE}

When someone suffers a traumatic spinal cord injury, injured tissue at the lesion site releases an excessive amount of a chemical called adenosine triphosphate (ATP) that activates a receptor called P2X7. The activation of P2X7 receptors causes further irreversible tissue damage or secondary injury.

In 2004, Maiken Nedergaard and colleagues at the University of Rochester Medical Center (NY) showed that a compound that blocked the P2X7 receptors reduced spinal cord damage and improved motor function recovery in rats that had experienced spinal cord injury (Nat. Med. $10,821-827 ; 2004)$. This compound, however, is toxic and cannot cross the blood-brain barrier, so it has only limited clinical utility.

Nedergaard and her team have now tested another compound, called Brilliant blue G (BBG), that also blocks P2X7. BBG is cheap and non-toxic, crosses the brain-blood barrier and is very similar to FD\&C blue dye No. 1, the compound used to dye Gatorade and M\&Ms blue. When given to rats with spinal cord injuries, BBG reduced tissue damage and improved motor recovery (Proc. Natl. Acad. Sci. USA $106,12489-12493 ; 2009)$.

To evaluate $B B G$, the research team anesthetized the rats and gave the rats a weight drop injury in the thoracic region of the spinal cord. They then injected the rats with either a control solution or a solution of BBG (at a dose of $10 \mathrm{or} 50 \mathrm{mg} / \mathrm{kg}$ ) 10-15 min after injury and once daily for the next two days. The researchers monitored motor recovery and injury size for the six weeks following injury.

The rats that received one of the BBG doses recovered motor function faster than did the control animals. From 10 through 42 days after injury (the end of the experiment), the BBG rats had significantly better motor function than the non-treated rats. Additionally, the rats that received either dose of BBG lost significantly less spinal cord tissue after the injury than did the rats treated with the control solution. Importantly, the BBG injections had a greater effect on mitigating tissue atrophy than on suppressing lesion size, which fits with the hypothesis that BBG blocks the response that causes secondary injury.

The only noticeable side effect was that the eyes of the rats that received either of the BBG doses and the skin of the rats that received the $50 \mathrm{mg} / \mathrm{kg} \mathrm{BBG}$ dose became temporarily blue. Though these results are promising, more extensive research is needed to determine whether this therapy could be useful for humans. Kirsten Dorans 